ARTICULO DE INVESTIGACION

\title{
Crecimiento en diámetro de Zanthoxylum kellermanii P. Wilson en una selva perennifolia del norte de Oaxaca, México
}

\author{
Diameter growth of Zanthoxylum kellermanii P. Wilson \\ in a tropical evergreen forest of northern Oaxaca, Mexico
}

\author{
Filemón Manzano-Méndez¹, Juan Ignacio Valdez-Hernández1 \\ Miguel Ángel López-López¹ y Humberto Vaquera-Huerta²
}

\begin{abstract}
RESUMEN
Se determinó el crecimiento estacional en diámetro de una especie de importancia maderable: Zanthoxylum kellermanii P. Wilson. Se seleccionaron 22 árboles en seis categorías diamétricas y se les instalaron bandas dendrométricas para tomar lecturas bimensuales de su crecimiento. También se registraron características relacionadas con la copa de los árboles: calidad (simetría, vigor, densidad del follaje) y posición (incidencia de luz). El crecimiento promedio anual en diámetro fluctuó de 0,86 $\mathrm{cm}$ a $1,87 \mathrm{~cm}$ en las categorías diamétricas, de $0,52 \mathrm{~cm}$ a $2,04 \mathrm{~cm}$ en las calidades de copa y de 0,73 $\mathrm{cm}$ a $1,70 \mathrm{~cm}$ en las posiciones de copa. Los análisis de varianza mostraron diferencias altamente significativas $(p<0,001)$ entre categorías diamétricas, así como entre calidades y posiciones de copa. Herramientas como la banda dendrométrica apoyada en modelos de regresión fueron de gran utilidad para el registro y proyección del incremento en diámetro de la especie estudiada.
\end{abstract}

PALABRAS CLAVE:

Banda dendrométrica, calidad y posición de copa, categoría diamétrica, especie maderable, tasa de crecimiento.

\begin{abstract}
The seasonal diameter growth of a commercially valuable tree species, Zanthoxylum kellermanii (P. Wilson), was determined. Twenty-two trees were selected in six diameter categories and dendrometric bands were used to take bimonthly growth readings. Other readings related to crown characteristics were also taken: quality (symmetry, vigor, density of foliage) and positions (light incidence). Mean annual growth in diameter fluctuated from $0,86 \mathrm{~cm}$ to $1,87 \mathrm{~cm}$ within diameter categories; from $0,52 \mathrm{~cm}$ to $2,04 \mathrm{~cm}$ within crown qualities and from $0,73 \mathrm{~cm}$ to $1,70 \mathrm{~cm}$ within crown positions. Analyses of variance showed highly significant differences $(p \leq 0,001)$ among diameter categories, as well as among crown qualities and positions. Tools such as dendrometric bands along with regression models, proved to be useful to determine and project the diameter increment of the species studied.
\end{abstract}

KEY WORDS:

Dendrometric band, crown quality and position, diameter category, timber species, growth rate. 


\section{INTRODUCCIÓN}

La aplicación de sistemas silviculturales basados en un manejo sostenible del bosque requiere información detallada de los patrones de crecimiento de los árboles en regiones tanto templadas como tropicales (Makocki y Valdez, 2001). Particularmente en México, estas últimas no han recibido una atención suficiente; aún cuando existen algunas proyecciones para especies como Swietenia macrophylla King o Cedrela odorata L. (Ricker y Del Río, 2004), para la mayoría de especies arbóreas en bosques tropicales no existe información científica de su crecimiento y rendimiento en madera. Zanthoxylum kellermanii $\mathrm{P}$. Wilson es una especie muy apreciada en zonas cálido-húmedas para construcción de casas (Pennington y Sarukhán, 2005) y fabricación de muebles, sus hojas y corteza contienen propiedades anti-inflamatorias y analgésicas (Lima et al., 2007).

La información del crecimiento radial se emplea para explicar cambios en diámetro y evaluar tasas de producción de madera en diferentes categorías a través del tiempo (Worbes, 1995). La medición del diámetro se utiliza para estimar, a través del uso de modelos alométricos variables de difícil medición, tales como biomasa de madera y foliar entre otros. En diversos estudios sobre silvicultura, ecología del bosque y fisiología de árboles se han utilizado una variedad de instrumentos de medición que a menudo difieren en exactitud, precisión, costo o simplicidad operacional (López et al., 2006). Un instrumento que se puede emplear es la banda dendrométrica, la cual tuvo sus inicios con Hall (1944) y se puede construir con cintas de acero inoxidable o aluminio. Liming (1957) sugiere una técnica que registre cambios en circunferencia mediante una escala sobrepuesta a la banda metálica, mientras que Cattelino et al. (1986) proporcionan detalles de su construcción, instalación y material utilizado. El empleo de bandas dendrométricas en especies arbóreas tropicales va en aumento (Pereira da Silva et al., 2002) y se está convirtiendo en una herramienta popular en varios aspectos de la investigación forestal (Galán, 2007). Particularmente en México, existen trabajos donde esta técnica no destructiva ha generado resultados satisfactorios (Makocki y Valdez, 2001; López et al., 2006). Por lo anterior y con el propósito de contribuir a un mejor manejo de la selva perennifolia en el norte del estado de Oaxaca, México, el presente trabajo tuvo como finalidad determinar las tasas de crecimiento anual en diámetro de Zanthoxylum kellermanii, una especie de importancia maderable en la región.

\section{OBJETIVO}

Comparar el crecimiento en diámetro de Zanthoxylum kellermanii P. Wilson entre categorías diamétricas, calidades y posiciones de copa, así como determinar sus tasas de crecimiento anual.

\section{METODOLOGÍA}

\section{Área de estudio}

Se localiza en San Martín Soyolapan, municipio de Santiago Comaltepec, ubicado al norte del estado de Oaxaca, México, en la región denominada Chinantla Alta $\left(17^{\circ} 41^{\prime} 53,5^{\prime \prime} \mathrm{N}\right.$; $96^{\circ} 16^{\prime}$ 57,3" O; $60 \mathrm{msnm}$ ).

El clima se clasifica como $A m$ e(g)w", cálido húmedo con lluvias en verano, con una temperatura media anual de $24{ }^{\circ} \mathrm{C}$ (máxima $28^{\circ} \mathrm{C}$, mínima $21^{\circ} \mathrm{C}$ ) y $3590 \mathrm{~mm}$ de precipitación al año (García, 1987). El tipo de vegetación corresponde a una selva alta perennifolia (Pennington y Sarukhán, 2005). 


\section{Selección de individuos y colocación de bandas dendrométricas}

En una superficie aproximada de cinco hectáreas, al borde del bosque natural, donde la especie de interés presentó una mayor densidad de individuos, se escogieron 22 árboles sanos y de fuste recto y concéntrico. Estos individuos se clasificaron en seis categorías (cat) diamétricas de acuerdo con Corella et al. (2001): 2,5 cm-7,5 cm (cat 5); 7,6 cm-12,5 cm (cat 10); $12,6 \mathrm{~cm}-17,5 \mathrm{~cm}$ (cat 15); $17,6 \mathrm{~cm}$ $22,5 \mathrm{~cm}$ (cat 20); 22,6 cm-27,5 cm (cat 25) y $27,6 \mathrm{~cm}-32,5 \mathrm{~cm}$ (cat 30 ), instalándoles bandas dendrométricas, siguiendo la metodología descrita por Cattelino et al. (1986), a una altura de 1,30 m sobre el nivel del suelo (DAP) para tomar lecturas bimensuales de su crecimiento. Las categorías de 20, 25 y $30 \mathrm{~cm}$ fueron evaluadas durante un periodo de 12 bimestres, mientras que las de 5,10 y 15 $\mathrm{cm}$ durante siete bimestres; esto debido a que en el rodal elegido no se encontraron las últimas tres categorías y se buscaron posteriormente en un rodal contiguo al área principal de estudio. Los individuos también se clasificaron con base en su calidad y forma de copa: buena (3), copa simétrica y vigorosa; regular (2), defectos en simetría y mediana densidad de hojas; deficiente (1), copa fracturada, asimétrica y poco vigorosa. Además se clasificaron de acuerdo con su posición de copa: expuesta (3), con entradas verticales y laterales de luz solar; semiexpuesta (2), con entradas parciales verticales o laterales de luz; y cubierta (1), con nula o muy poca entrada de luz solar (adaptado de Dawkins, 1958).

\section{Análisis estadístico}

Con los datos de crecimiento obtenidos bimensualmente se realizaron análisis de regresión no lineal con el paquete estadístico Curve expert versión 1.3 (2008) para modelar el crecimiento acumulado en función del tiempo. Para ajustar la base de datos se probaron los siguientes modelos: cuadrático (1): $\mathrm{y}=b_{0}+b_{1} \mathrm{x}+b_{2} \mathrm{x}^{2}$, exponencial (2): $\mathrm{y}=b_{0}\left[1-\exp \left(-b_{1} \mathrm{x}\right)\right]$ y logístico (3): $\mathrm{y}=b_{0} /\left[1+b_{1} \exp \left(-b_{2} \mathrm{x}\right)\right]$, evaluados a través de sus valores estadísticos como el cuadrado medio del error, $R^{2}$, y nivel de significancia de los modelos, seleccionando el modelo exponencial por su sencillez y por presentar un mejor ajuste. Una vez obtenidas las ecuaciones de cada individuo en las categorías diamétricas, así como en las clases de calidad y posición de copa, se calcularon valores con el modelo ajustado y se obtuvieron curvas de crecimiento tanto individuales como promedio, las cuales se representaron gráficamente. Con los datos observados se realizaron análisis de varianza y pruebas de comparación de medias (Tukey, $p \leq 0.05$ ) empleando el paquete estadístico SAS versión 8.0 (SAS Institute, 1999). Con los datos obtenidos de los modelos de regresión ajustados por categoría diamétrica, calidad de copa y posición de copa, se calcularon las tasas de crecimiento promedio por bimestre y la del incremento promedio anual. Finalmente se correlacionaron los valores de calidad con los de posición de copa mediante la utilización de la prueba de Spearman.

\section{RESULTADOS Y DISCUSIÓN}

\section{Categorías diamétricas}

El crecimiento acumulado en diámetro para los 22 árboles seleccionados se muestra en la figura 1. Los crecimientos promedio anuales fluctuaron de $0,86 \mathrm{~cm}$ a $1,87 \mathrm{~cm}$ entre las diversas categorías diamétricas; $0,86 \mathrm{~cm}$ corresponde a la categoría diamétrica de 20 y $1,87 \mathrm{~cm}$ a la categoría 10. Sin embargo, fue posible encontrar individuos que crecieron hasta $3,11 \mathrm{~cm} / a n ̃ o$ (Tabla 1). 

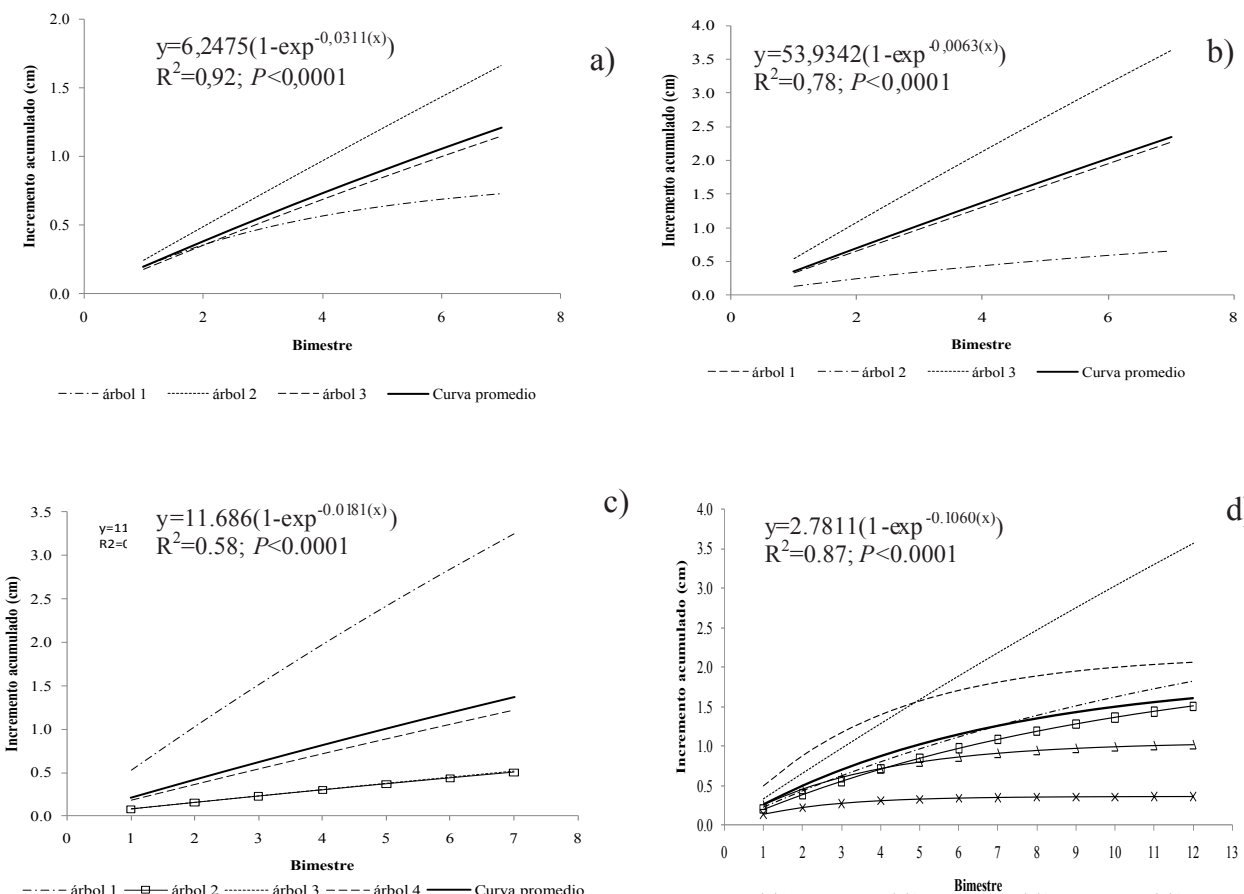

c)

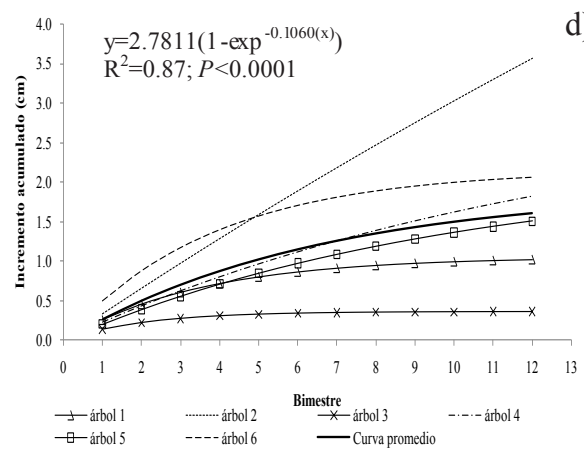

e)
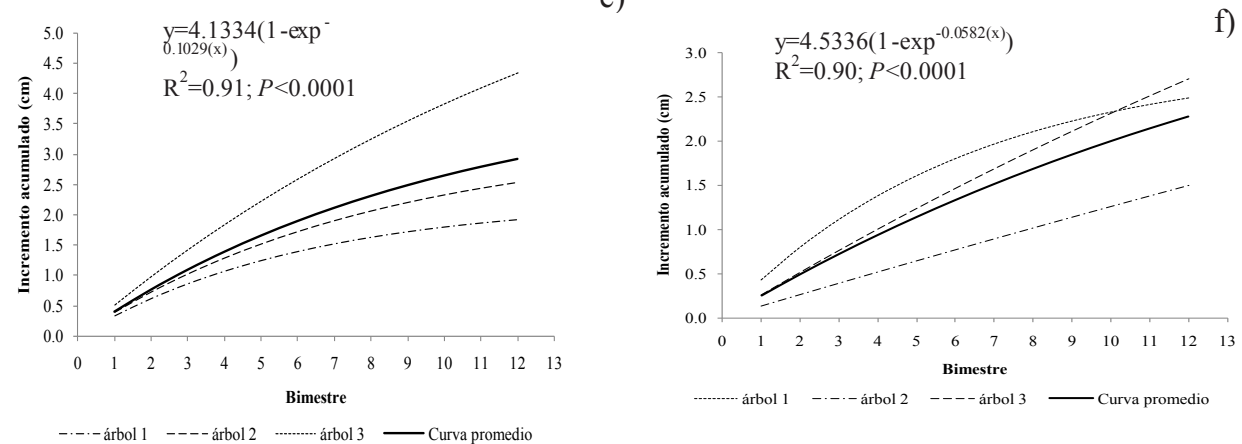

Figura 1. Crecimiento acumulado en diámetro para árboles de Zanthoxylum kellermanii por categoría diamétrica: $5 \mathrm{~cm}$ (a), $10 \mathrm{~cm}$ (b), $15 \mathrm{~cm}$ (c), $20 \mathrm{~cm}$ (d), $25 \mathrm{~cm}$ (e) y $30 \mathrm{~cm}$ (f). La ecuación corresponde a la curva promedio en cada categoría. 
Tabla 1. Crecimiento promedio anual en diámetro mínimo, medio y máximo de Zanthoxylum kellermanii por categoría diamétrica.

\begin{tabular}{cccccc}
\hline Categoría diamétrica & $\begin{array}{c}\text { Número } \\
\text { de árboles }\end{array}$ & \multicolumn{2}{c}{$\begin{array}{c}\text { Crecimiento promedio anual } \\
\text { en diámetro (cm/año) }\end{array}$} & $\begin{array}{c}\text { Desviación } \\
\text { estándar }\end{array}$ \\
\cline { 3 - 4 } 5 & 3 & 0,6238 & 1,0094 & 1,4209 & 0,3992 \\
10 & 3 & 0,5622 & 1,8708 & 3,1109 & 1,2757 \\
15 & 4 & 0,4293 & 1,1746 & 2,7789 & 1,1070 \\
20 & 6 & 0,1802 & 0,8629 & 1,7875 & 0,5458 \\
25 & 3 & 0,9608 & 1,4676 & 2,1740 & 0,6301 \\
30 & 3 & 0,7510 & 1,1162 & 1,3535 & 0,3202 \\
\hline
\end{tabular}

Estos valores son similares al observado por Pereira et al. (2002) para una región natural en la Amazonía Central de Brasil $(1,64 \mathrm{~cm})$, pero menores al encontrado por Russo y Sandí (1995) para la misma especie en la región tropical húmeda de Costa Rica $(5,12 \mathrm{~cm})$ aunque en condiciones de plantación y bajo manejo, lo cual puede explicar esta diferencia en crecimiento. Al respecto, Foli et al. (2003) sostienen que la densidad del rodal y el espacio de crecimiento en los trabajos de plantación representan una guía útil para la práctica silvicultural en bosques tropicales.
Los resultados del análisis de varianza muestran que existen diferencias altamente significativas ( $p<0,001)$ entre categorías diamétricas y entre bimestres de medición (Tabla 2).

El valor medio del crecimiento en diámetro de la categoría 10 fue significativamente mayor que las categorías restantes (Tukey, $p<0,05$ ), de la misma manera la categoría 25 con respecto a las 15 y 5 , mientras que las categorías 25,20 y 30 fueron estadísticamente similares (Tukey, $p>0,05)$, al igual que las 20,30, 15 y 5 (Tabla 3 , Figura 2).

Tabla 2. Análisis de varianza del crecimiento en diámetro por categoría diamétrica registrado durante siete bimestres en árboles de Zanthoxylum kellermanii.

\begin{tabular}{lccc}
\hline FUENTE DE VARIACIÓN & g.I. & CM & Pr>F \\
\hline Categorías diamétricas & 5 & 0,368 & $<0,0001$ \\
Bimestres & 6 & 1,4667 & $<0,0001$ \\
Error & 30 & 0,019 & \\
\hline
\end{tabular}

g.I.= grados de libertad; $\mathrm{CM}=$ cuadrados medios; $\mathrm{Pr}>\mathrm{F}=$ significancia 
Tabla 3. Valores medios del crecimiento en diámetro por categoría diamétrica para árboles de Zanthoxylum kellermanii.

\begin{tabular}{ccc}
\hline CATEGORIA & N & MEDIA \\
\hline 10 & 7 & $1,36129 \mathrm{a}$ \\
25 & 7 & $1,09057 \mathrm{~b}$ \\
20 & 7 & $0,92000 \mathrm{bc}$ \\
30 & 7 & $0,91657 \mathrm{bc}$ \\
15 & 7 & $0,80957 \mathrm{c}$ \\
5 & 7 & $0,71671 \mathrm{c}$ \\
DMS & & 0,2247 \\
\hline
\end{tabular}

Valores con letras distintas fueron estadísticamente diferentes (Tukey, $\mathrm{p}<0,05$ ); $\mathrm{N}=$ bimestres; DMS = diferencia mínima significativa

El comportamiento puede ser explicado con base en el crecimiento alternado de la altura y grosor del tallo en las diferentes categorías diamétricas. Así, las categorías intermedias (p.e. cat 10) parecen destinar recursos para aumentar en diámetro (Klepac, 1983), mientras las categorías pequeñas (p.e. cat 5) tienen un crecimiento lento (Diéguez et al., 2003), el cual se refleja en una menor pendiente de la curva de crecimiento (Louman et al., 2001). Sin embargo, estas categorías pequeñas pueden estar aumentando en altura. Al respecto, Harold y Hocker (1984) mencionan que en la mayoría de las especies la tasa de crecimiento en altura es mayor que los incrementos en diámetro en las primeras etapas de desarrollo. En cambio, las categorías grandes (p.e. cat 30) van disminuyendo su crecimiento gradualmente, llegando a ser muy reducido (Klepac, 1983; Wadsworth, 2000).

\section{Calidad y posición de copa}

En la tabla 4 se observan los crecimientos diamétricos mínimos, medios y máximos, mientras que el crecimiento acumulado por calidad de copa se muestra en las figuras 3 a 5 .

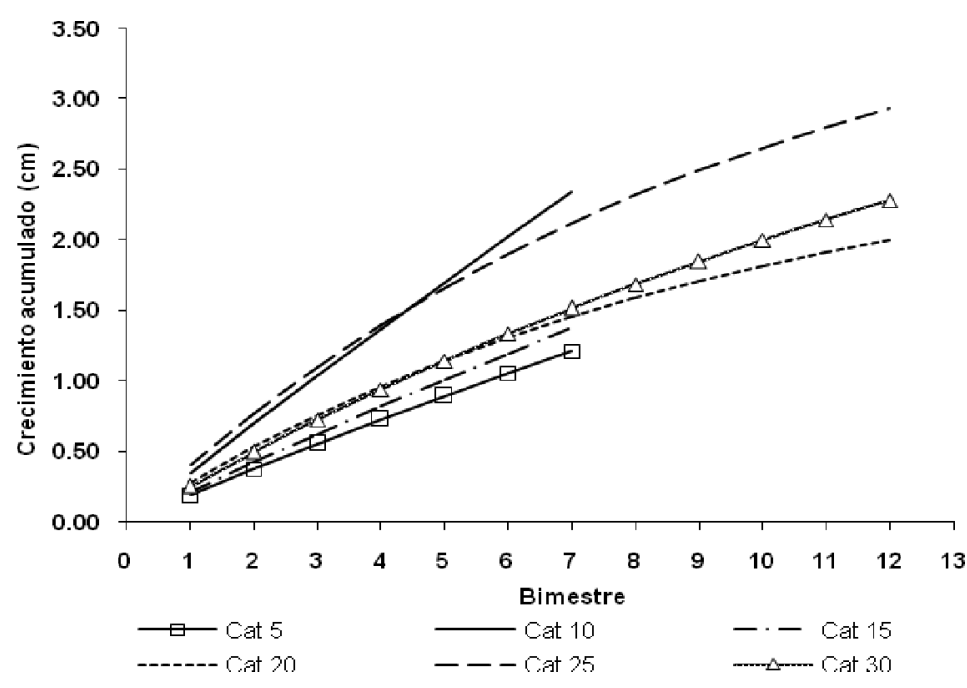

Figura 2. Crecimiento promedio acumulado en diámetro para árboles de Zanthoxylum kellermanii por categoría diamétrica. 
Tabla 4. Crecimiento promedio anual en diámetro mínimo, medio y máximo de Zanthoxylum kellermanii por calidad de copa.

\begin{tabular}{|c|c|c|c|c|c|}
\hline \multirow[t]{2}{*}{ Calidad de copa } & \multirow[t]{2}{*}{$\begin{array}{l}\text { Número } \\
\text { de árboles }\end{array}$} & \multicolumn{3}{|c|}{$\begin{array}{c}\text { Crecimiento promedio anual } \\
\text { en diámetro (cm/año) }\end{array}$} & \multirow[t]{2}{*}{$\begin{array}{l}\text { Desviación } \\
\text { estándar }\end{array}$} \\
\hline & & Mínimo & Medio & Máximo & \\
\hline 3 (buena) & 7 & 1,0348 & 2,0352 & 3,1108 & 0,7278 \\
\hline 2 (regular) & 9 & 0,5092 & 1,0032 & 1,3535 & 0,2668 \\
\hline 1 (deficiente) & 6 & 0,2990 & 0,5210 & 0,7668 & 0,1649 \\
\hline
\end{tabular}

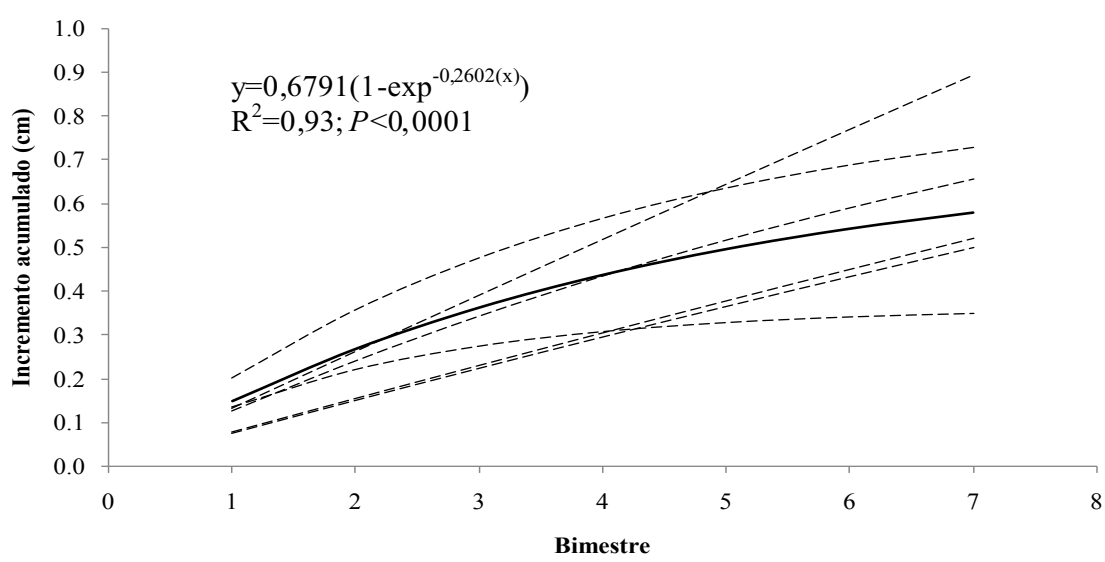

Figura 3. Crecimiento acumulado en diámetro para árboles $(n=6)$ de Zanthoxylum kellermanii con calidad de copa uno. La ecuación corresponde a la curva promedio (línea continua).

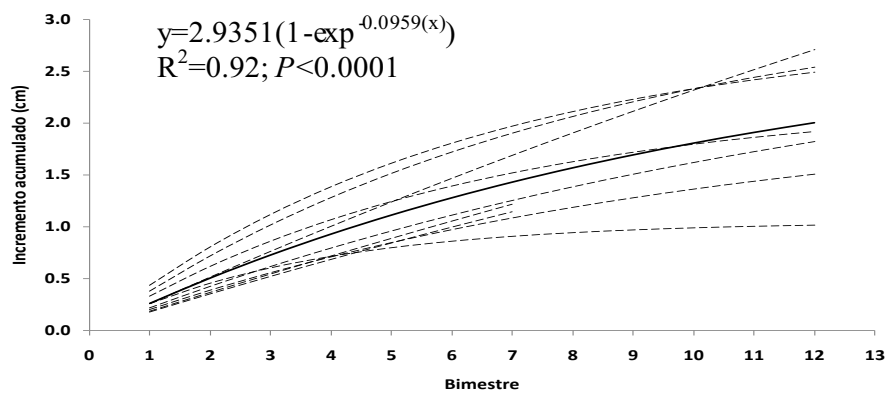

Figura 4. Crecimiento acumulado en diámetro para árboles $(n=9)$ de Zanthoxylum kellermanii con calidad de copa dos. La ecuación corresponde a la curva promedio (línea continua). 


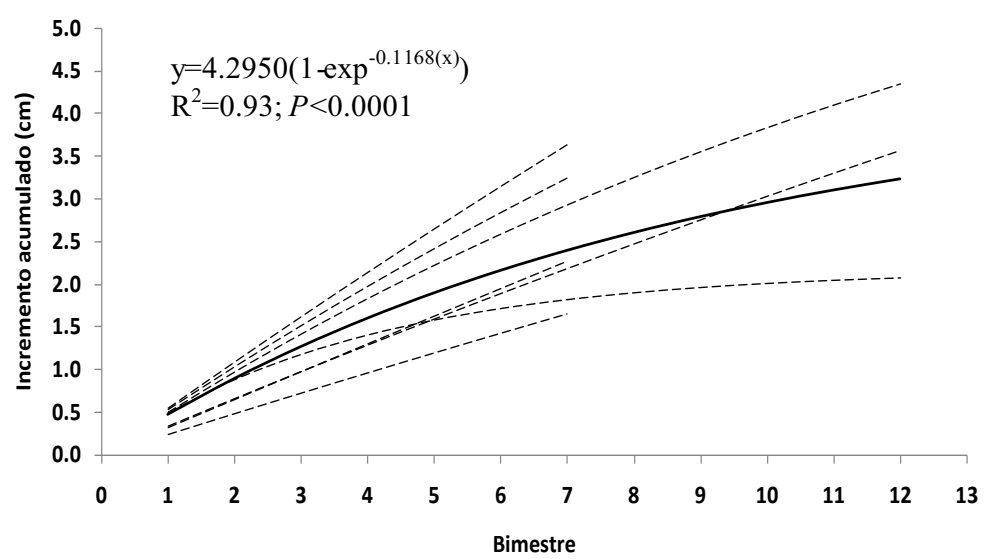

Figura 5. Crecimiento acumulado en diámetro para árboles $(n=7)$ de Zanthoxylum kellermanii con calidad de copa tres. La ecuación corresponde a la curva promedio (línea continua).

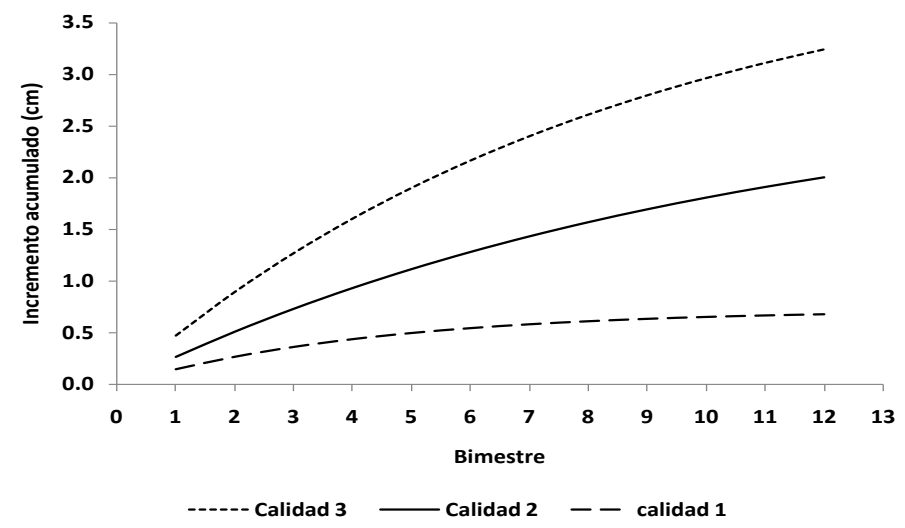

Figura 6. Crecimiento promedio acumulado en diámetro para árboles de Zanthoxylum kellermanii por calidad de copa.

Las curvas promedio del crecimiento acumulado en diámetro para árboles clasificados en tres calidades de copa se observan en la figura 6 .

Los valores del crecimiento en diámetro mostraron diferencias altamente significativas $(p<0,001)$ entre calidades de copa y bimestres de registro de las mediciones (Tabla 5). Este resultado se apoya en que el aspecto o calidad de copa se relaciona con el tamaño y estado de desarrollo del árbol, así como con su incremento potencial (Dawkins, 1963; O'Brien et al., 1995; Sterck y Bongers, 2001), y que el diámetro y conformación de la copa tienen una relación positiva con el diámetro del fuste (Smith et al., 1992; Alves y Santos, 2002; Foli et al., 2003). 
Tabla 5. Análisis de varianza del crecimiento en diámetro por calidad de copa para árboles de Zanthoxylum kellermanii.

\begin{tabular}{lccc}
\hline FUENTE DE VARIACIÓN & g.l. & CM & Pr>F \\
\hline Calidades de copa & 2 & 2,2215 & $<0,0001$ \\
Bimestres & 6 & 0,5360 & $<0,0001$ \\
Error & 12 & 0,7292 & \\
\hline
\end{tabular}

g.I. = grados de libertad; $\mathrm{CM}=$ cuadrados medios; $\mathrm{Pr}>\mathrm{F}=$ significancia

Los valores medios del crecimiento en diámetro para las calidades de copa fueron significativamente diferentes (Tukey, $p<0,05)$, (Tabla 6), observándose un mayor crecimiento para los individuos de calidad 3, intermedio para los de calidad 2 y menor para los de calidad 1. Al respecto, Swaine et al. (1987) sugieren que los árboles con mejor conformación estructural de la copa tienden a crecer más en diámetro, mientras que Louman et al. (2001) señalan que la forma e integridad de la copa es un buen indicador del vigor del árbol.

Con respecto a la categorización por posición de copa, los individuos con posición 3 tuvieron el mayor crecimiento promedio anual que los clasificados como posición 2. (Tabla 7).
El crecimiento acumulado en diámetro de los árboles clasificados por posición de copa se muestra en las figuras 7 y 8 .

Tabla 6. Valores medios del crecimiento en diámetro por calidad de copa para árboles de Zanthoxylum kellermanii.

\begin{tabular}{ccc}
\hline CALIDAD & $\mathrm{N}$ & MEDIA \\
\hline 3 & 7 & $1,5291 \mathrm{a}$ \\
2 & 7 & $0,8980 \mathrm{~b}$ \\
1 & 7 & $0,4053 \mathrm{c}$ \\
DMS & & 0,3851 \\
\hline
\end{tabular}

Valores con letras distintas fueron estadísticamente diferentes (Tukey, $p<0,05$ ); $\mathrm{N}=$ bimestres; $\mathrm{DMS}=$ diferencia mínima significativa.

Tabla 7. Crecimiento promedio anual en diámetro mínimo, medio y máximo de Zanthoxylum kellermanii por posición de copa.

\begin{tabular}{|c|c|c|c|c|c|}
\hline \multirow[t]{2}{*}{ Posición de copa } & \multirow[t]{2}{*}{$\begin{array}{l}\text { Número } \\
\text { de árboles }\end{array}$} & \multicolumn{3}{|c|}{$\begin{array}{l}\text { Crecimiento promedio anual } \\
\text { en diámetro (cm/año) }\end{array}$} & \multirow[t]{2}{*}{$\begin{array}{l}\text { Desviación } \\
\text { estándar }\end{array}$} \\
\hline & & Mínimo & Medio & Máximo & \\
\hline 3 & 11 & 0,9131 & 1,7027 & 3,1108 & 0,7387 \\
\hline 2 & 11 & 0,4293 & 0,7370 & 1,2678 & 0,2811 \\
\hline
\end{tabular}




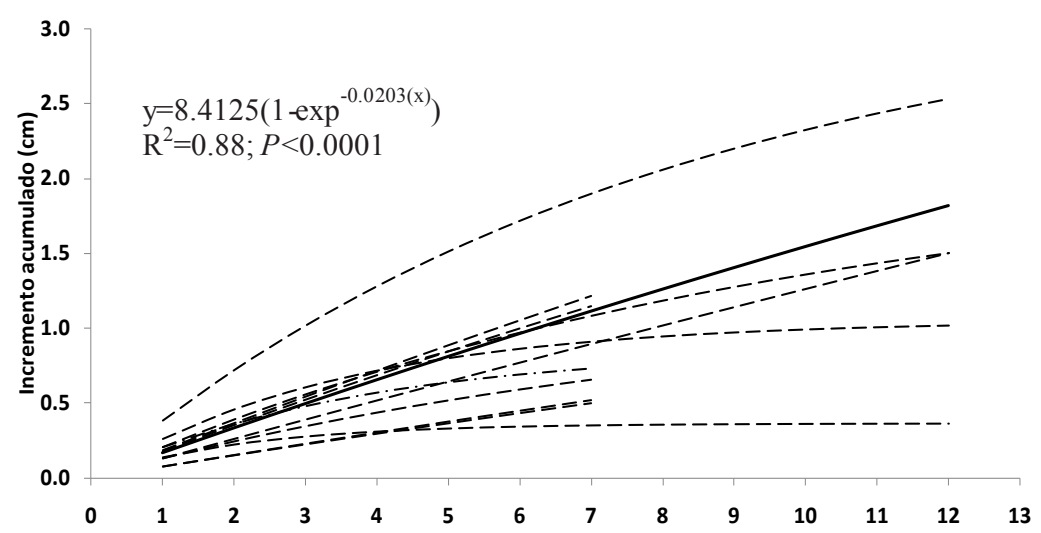

Figura 7. Crecimiento acumulado en diámetro para árboles $(n=11)$ con posición de copa dos. La ecuación corresponde a la curva promedio (línea continua).

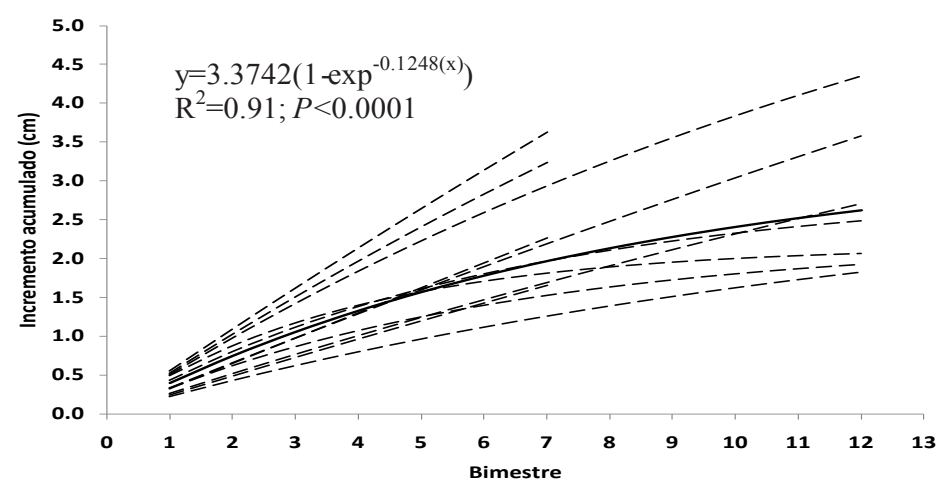

Figura 8. Crecimiento acumulado en diámetro para árboles $(n=11)$ con posición de copa tres. La ecuación corresponde a la curva promedio (línea continua).

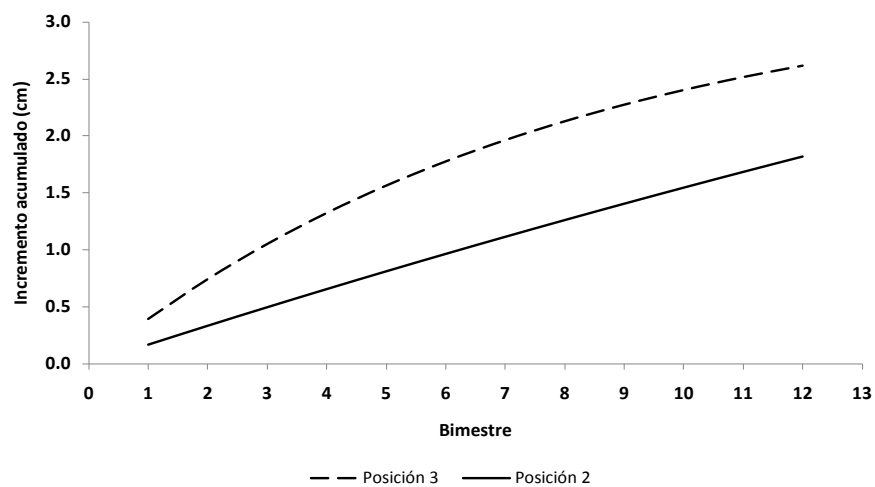

Figura 9. Crecimiento promedio acumulado en diámetro para árboles de Zanthoxylum kellermanii por posición de copa. 
Tabla 8. Análisis de varianza del crecimiento en diámetro por posición de copa registrado para árboles de Zanthoxylum kellermanii.

\begin{tabular}{lccc}
\hline FUENTE DE VARIACIÓN & g.I. & CM & Pr>F \\
\hline Posiciones de copa & 1 & 0,4810 & $<0,0001$ \\
Bimestres & 11 & 0,3646 & $<0,0001$ \\
Error & 23 & 0,0150 & \\
\hline
\end{tabular}

g.I. = grados de libertad; $\mathrm{CM}=$ cuadrados medios; $\mathrm{Pr}>\mathrm{F}=$ significancia

Las curvas promedio del crecimiento acumulado en diámetro para árboles clasificados en dos posiciones de copa se observan en la figura 9.

Los valores del crecimiento en diámetro revelaron diferencias altamente significativas $(p<0,001)$ entre posiciones de copa y bimestres de registro de las mediciones (Tabla 8).

La media del crecimiento en diámetro para la posición de copa tres fue significativamente (Tukey, $p<0,05$ ) mayor a la de la posición de copa dos (Tabla 9), lo cual sugiere que individuos con recepción directa de luz solar tienen un mayor crecimiento diamétrico (Silva et al., 1995; Hubbell et al., 1999). Al respecto, Clark y Clark (1999) y Louman et al. (2001) mencionan que la posición relativa de copa en el dosel determina su acceso a la luz, lo que a su vez, implica diferencias en crecimiento de individuos para una misma población.

Considerando lo anterior, muchas especies en bosques tropicales requieren para su crecimiento y reproducción de luz solar directa, por lo que muestran respuestas positivas en su crecimiento a las aperturas en el dosel (Denslow, 1987; Clark y Clark, 1994). Así mismo Camacho y Finegan (1997) señalan que la exposición a la luz representa una de las varia- bles silviculturales de mayor relación con el incremento diamétrico.

Desde un punto de vista sucesional, Zanthoxylum kellermanii es considerada como una especie pionera de rápido crecimiento (Brokaw, 1985), la cual es favorecida por una alta iluminación, ocupando espacios creados por perturbaciones (Lozada y Arends, 2000) y como regeneración natural en zonas bajo aprovechamiento maderable (Grela, 2003).

Tabla 9. Valores medios del crecimiento en diámetro por posición de copa para árboles de Zanthoxylum kellermanii.

\begin{tabular}{ccc}
\hline POSICIÓN & N & MEDIA \\
\hline 3 & 7 & $1,02043 \mathrm{a}$ \\
2 & 7 & $0,64971 \mathrm{~b}$ \\
DMS & & 0,1606 \\
\hline
\end{tabular}

Valores con letras distintas fueron estadísticamente diferentes (Tukey, $\mathrm{p}<0,05$ ); $\mathrm{N}=$ bimestres; $\mathrm{DMS}=$ diferencia mínima significativa.

La calidad y posición de la copa se correlacionaron positivamente $(r=0,95 ; p<$ 0,0001 ), lo cual indica que la calidad estuvo significativamente asociada con la posición que tiene el árbol en el dosel; al respecto, Zamudio-Sánchez y López- 
Torres (1999) señalan que la superficie expuesta de la copa del árbol puede considerarse una medida del grado del vigor de los individuos en un sitio, mientras que Clark y Clark (1999) mencionan que la posición de la copa tiene importancia para el vigor del árbol y su calidad de copa, determinando diferencias en crecimiento.

\section{CONCLUSIONES}

El modelo que mejor describe el crecimiento acumulado de Zanthoxilum kellermanii en función del tiempo es el modelo exponencial, el cual representa a su vez un modelo sencillo y de fácil aplicación para la especie. Existieron diferencias significativas de crecimiento en diámetro para árboles de Zanthoxylum kellermanii entre categorías diamétricas, así como entre calidades y posiciones de copa. Los mayores crecimientos diamétricos anuales para esta especie se registraron en individuos de 7,6 a $12,5 \mathrm{~cm}$ (categoría diamétrica 10), con buena calidad de copa (simétrica, vigorosa) y expuestas, tanto vertical como lateralmente a la luz solar. De acuerdo con los resultados obtenidos, Zanthoxylum kellermanii es una especie considerada de sucesión temprana, susceptible de ser utilizada en programas de recuperación y enriquecimiento de áreas perturbadas en la zona de estudio.

\section{AGRADECIMIENTOS}

A la fundación Ford y al Programa de Becas de Postgrado para Indígenas de México por la beca otorgada al primer autor para cursar el doctorado en el Postgrado Forestal del Colegio de Postgraduados; a Elizandro Pineda Herrera por su apoyo en el trabajo de campo y a Víctor Manuel Interián Ku y Esmeralda Cázares Sánchez por la ayuda en la interpretación estadística.

\section{REFERENCIAS}

Alves, L.F. y F.A.M. Santos. 2002. Tree allometry and crown shape of four tree species in Atlantic rain forest, Southeast Brazil. Journal of Tropical Ecology 18(2):245-260.

Brokaw, N.V.L. 1985. Gap-phase regeneration in a tropical forest, Ecology 66(3):682-687.

Camacho, M. y B. Finegan. 1997. Efectos del aprovechamiento forestal y el tratamiento silvicultural en un bosque húmedo del noreste de Costa Rica. Centro Agronómico Tropical de Investigación y Enseñanza, CATIE. Informe técnico No. 295. Colección Manejo diversificado de Bosques Naturales. No $11.38 \mathrm{p}$.

Cattelino, P.J., C.A. Becher y L.G. Fuller. 1986. Construction and installation of homemade dendrometer bands. Northern Journal of Applied Forestry 3:73-75.

Clark, D.A. y D.B. Clark. 1994. Climateinduced annual variation in canopy tree growth in a Costa Rican tropical rain forest. Journal of Ecology 82:865-872.

Clark, D.A. y D.B. Clark. 1999. Assessing the growth of tropical rain forest trees: issues for forest modeling and management. Ecological applications 9(3):981-997.

Corella, J.F., J.I. Valdez H., V.M. Cetina A., F. González C., A. Trinidad S. y J.R. Aguirre. 2001. Estructura forestal de un bosque de mangles en el noreste del estado de Tabasco, México. Revista Ciencia Forestal en México 25(90):73-101.

Curve expert 1.3. 2008. (en línea). Disponible en www.ebicom.net dhyams/ cftf.htm. Revisado el 5 de abril de 2008. 
Da Silva, R.P., J. Dos Santos, E. Siza T., J.Q. Chambers, S. Nakamura y N Higuchi. 2002. Diameter increment and growth patterns for individual tree growing in Central Amazon, Brazil. Forest Ecology and Management 166:295-301.

Dawkins, H.C. 1958. The management of natural tropical high forest with special reference to Uganda. Oxford University Press, Oxford. Imperial Forestry Institute. Paper No. 34. 155 p.

Dawkins, H.C. 1963. Crown diameters: their relationship to bole diameter in tropical trees. Forest Review 42(4):318-333.

Denslow, J.S. 1987. Tropical rainforest gaps and tree species diversity. Annual Review of Ecology and Systematics 18:431-451.

Diéguez, A.U., M. Barrio A., F. Castedo D., A.D. Ruíz G., M.F. Álvarez T., J.G. Álvarez y A. Rojo. 2003. Dendrometría. Ediciones MundiPrensa. Madrid, España. 327 p.

Foli, E.G., D. Alder, H.G. Miller y M.D. Swaine. 2003. Modelling growing space requirements for some tropical forest tree species. Forest Ecology and Management 173:79-88.

Galán, L.R. 2007. Crecimiento y rendimiento de especies arbóreas en una plantación de enriquecimiento de acahuales en el sur de Oaxaca. Tesis de Maestría. Programa Forestal. Colegio de Posgraduados. Montecillo. México. 155 p.

García, M.E. 1987. Modificaciones al sistema de clasificación climática de Köppen. Cuarta edición. México, D.F. 217 p.
Grela, I.A. 2003. Evaluación del estado sucesional de un bosque subtropical de quebradas en el norte de Uruguay. Acta Botánica Brasileira 17(2):315-324.

Hall, R.C. 1944. A vernier tree-growth band. Journal of Forestry 42:742743.

Harold, W. y Hocker Jr. 1984. Introducción a la Biología Forestal. Primera edición en Español. Traducido por F.A. Bellomo López. AGT EDITOR S.A. México. 446 p.

Hubbell, S.P., R.B. Foster, S.T. O'Brien, K. Harms, R. Condit y B. Wechsler. 1999. Light-gap disturbances, recruitment limitation, and tree diversity in a Neotropical forest. Science 283:554-557.

Klepac, D. 1983. Crecimiento e incremento de árboles y masas forestales. $2^{\mathrm{a}}$. ed. Departamento de Enseñanza, Investigación y Servicio en Bosques. Universidad Autónoma Chapingo. Chapingo, México. 365 p.

Lima, L.M., P. Ferreira, C.J. Tavares y J.K. Bastos. 2007. Anti-inflamatory and analgesic activities of the ethanolic extracts from Zanthoxylum riedelianum (Rutaceae) leaves and stem bark. Journal of Pharmacy and Pharmacology 59(8):1151-1158.

Liming, F.G. 1957. Homemade dendrometers. Journal of Forestry 55:575577.

López, A.J.L., J.I. Valdez H., T. Terrazas y J.R. Valdez L. 2006. Crecimiento en diámetro de especies arbóreas en una selva mediana subcaducifolia en Colima, México. Agrociencia 40(1):139-147. 
Louman, B.,J. Valerio, W. Jiménez. 2001. Bases ecológicas. In: B. Louman, D. Quirós y M. Nilsson (editores). Silvicultura de bosques latifoliados húmedos con énfasis en América Central. Serie Técnica. Manual Técnico No 46. CATIE. Costa Rica. pp:19-78.

Lozada, J.R y E. Arends. 2000. Clasificación ecológica de especies, con fines de Aprovechamiento Forestal en la estación Caparo. Revista Forestal Venezolana 44(1):81-91.

Makocki, M.W. y J.I. Valdez H. 2001. Radial growth periodicity of tree species in a semi-deciduous tropical forest of the Pacific coast of México: Implications for their management. In: Proceedings of the IUFRO Joint Symposium on Tree Seed Technology, Physiology and Tropical Silviculture. College of Forestry and Natural Resources. University of the Philippines, Los Baños. pp:85-96.

O'Brien, S.T., S.P. Hubbell, P. Spiro, R. Condit y R.B. Foster. 1995. Diameter, height, crown, and age relationships in eigth neotropical tree species. Ecology 76(6):1926-1939.

Pennington, T.D. y J. Sarukhán K. 2005. Árboles tropicales de México: Manual para la identificación de las principales especies. Texto Científico Universitario. Tercera edición. Fondo de Cultura Económica. Universidad Nacional Autónoma de México. México, D.F. 523 p.

Pereira da Silva R., J. Dos Santos, E. Siza T., J.Q. Chambers, S. Nakamura y N. Higuchi. 2002. Diameter increment and growth patterns for individual tree growing in Central Amazonia, Brazil. Forest Ecology and Management 166:295-301.
Ricker, M. y R. Del Río. 2004. Projecting diameter growth in tropical trees: a new modelling approach. Forest Science 50(2):213-224.

Russo, R.O. y C.L. Sandí. 1995. Early growth of eight native timber species in the humid tropic region of Costa Rica. Journal of Sustainable Forestry 3(1):81-84.

SAS Institute Inc. 1999. User's guide, version 8.0 for Windows. Cary, NC. $595 \mathrm{p}$.

Silva, J.N.M., J.O.P. de Carvalho, J. C.A. do Lopes, B.F. de Almeida, D.H.M. Costa, L.C. de Oliveira, J.K. Vanclay y J.P. Skovsgaard. 1995. Growth and yield of a tropical rain forest in the Brazilian Amazon 13 years after logging. Forest Ecology and Management 71:267-274.

Smith, W.R., R.M. Farrar, P.A. Murphy, J.L. Yeiser, R.S. Meldahal y J.S. Kush. 1992. Crown and basal area relationships of open grown southern pines for modelling competition and growth. Canadian Journal of Forestry and Research 22:341-347.

Sterck, F.J. y F. Bongers. 2001. Crown development in tropical rain forest trees: patterns with tree height and light availability. Journal of Ecology 89(1):1-13.

Swaine, M.D., J.B. May y I.J. Alexander. 1987. Tree populations dynamics at Kade, Ghana (1968-1982). Journal of Tropical Ecology 3:331-345.

Wadsworth, H.F. 2000. Producción forestal para América Tropical. Departamento de Agricultura de los EE.UU. Servicio Forestal. Manual de Agricultura 710-S. Washington, DC. 563 p. 
Worbes, M. 1995. How to measure growth dynamics in tropical trees: a review. IAWA Journal 16:337-351.

Zamudio-Sánchez, F.J. y J.L. López-Torres. 1999. Estudio de la relación área mínima contra área real de la superficie de la copa en arboles de vegetación secundaria en Campeche, México. Revista Chapingo Serie Ciencias Forestales y del Ambiente 5(2): 167-171. selva perennifolia del norte de Oaxaca, México. Madera y Bosques 16(2):19-33. 